\title{
Proliferative Glomerulonephritis with Monoclonal IgG Deposits
}

National Cancer Institute

\section{Source}

National Cancer Institute. Proliferative Glomerulonephritis with Monoclonal Ig G Deposits. NCI Thesaurus. Code C158970.

A sub-type of monoclonal gammopathy of renal significance, characterized by restriction to a single immunoglobulin $\mathrm{G}$ heavy chain subclass and a single light chain isotype. Light microscopy often shows an endocapillary proliferative or membranoproliferative glomerulopathy. Electron microscopy reveals electron-dense subendothelial and mesangial deposits. 